\title{
JUSTICIA PARA INMIGRANTES: MERCADO Y POLÍTICA DE EXTRANJERÍA*
}

\author{
Ricard Zapata Barrero \\ Universidad Pompeu Fabra \\ E-mail: ricard.zapata@cpis.upf.es
}

\begin{abstract}
RESUMEN
Este artículo examina desde un punto de vista normativo la relación entre la justicia y la extranjería, en particular las cuestiones sobre los límites de la justicia en una política de extranjería. La pregunta que me he planteado es la siguiente: ¿es moralmente justo que los Estados legitimen sus políticas de extranjería en base a unos criterios económicos? El argumento que guiará mi razonamiento es que una política de extranjería tiene un carácter más justo cuando el mercado no funciona como criterio de orientación. En la primera parte presento, como preliminar, los nuevos escenarios del debate teórico político en torno a la relación entre el mercado y la justicia, con el fin de precisar el contexto de mi análisis. En la segunda parte introduzco brevemente las principales tensiones que ponen en duda la legitimidad del tratamiento estatal hacia los extranjeros desde el punto de vista de la justicia, con el propósito de presentar la lógica de mi razonamiento. En la tercera parte propongo una reflexión abierta, tomando la Ley de Extranjería espanoola como estudio de caso. En la última parte resumo mi argumento: la necesidad de revisar los criterios que legitiman las políticas de inmigración desde el punto de vista de la justicia en general, los criterios basados en el mercado en particular.
\end{abstract}

* Este artículo es la versión definitiva de un trabajo que defendí en la International Political Science Association-Research Committee on Political Philosophy (Rotterdam, junio 1998) bajo el tema Markets and Democracy. Agradezco los comentarios y críticas recibidas por B. Barber, F. Dallmayr, P. King, P. Lehning, P. Springborg, J. M. Rosales, S. Caney y Th. Hueglin, y en general a todos los panelistas y asistentes. 
El objetivo principal de este artículo es discutir la visión utilitarista de los inmigrantes que recorre tanto el discurso social como político y jurídico. El inmigrante no es ni un beneficio ni una carga para la sociedad receptora. Abordar la inmigración con esta lógica, aunque sea con buenas intenciones si se utiliza como beneficio, es vulnerar el más elemental principio de justicia de no tratar a las personas como medios sino como fines en sí mismas. Con este fin, este artículo examina desde un punto de vista normativo la relación entre la justicia y la inmigración, en particular las cuestiones sobre los límites de la justicia en una política de extranjería. La pregunta que me he planteado es la siguiente: ¡es justo que los Estados legitimen sus políticas de admisión sobre la base de unos criterios económicos? Mi intención es sugerir que la forma en que las políticas de extranjería tratan la relación entre el mercado y los extranjeros puede considerarse como un problema de justicia. Siguiendo esta dirección, el argumento que guiará mi razonamiento es que una política de extranjería tiene un carácter más justo cuando el mercado no funciona como criterio de orientación. Esta propuesta no tiene, evidentemente, un carácter absoluto. Existen otros implícitos que merecerían el mismo tratamiento. Las tensiones que resultan cuando se compara la justicia y la inmigración son complejas (W. F. Schwartz, ed., 1995). Lo que aquí defenderé es sólo un aspecto de esta problemática, como base para seguir explorando esta línea de trabajo.

\section{NUEVOS ESCENARIOS PARA REPLANTEAR LA RELACIÓN ENTRE EL MERCADO Y LA JUSTICIA EN ESTA PRIMERA DÉCADA DEL SIGLO XXI}

Actualmente existen al menos tres hechos históricos que proporcionan a la relación entre el mercado y la justicia un carácter distintivo: el problema del pluralismo cultural, el proceso de construcción política de una Unión Europea (UE) y, finalmente, la dinámica globalizadora en la que estamos inmersos. Estos fenómenos políticos pueden ser considerados como estando en el origen de tensiones que están en el epicentro de la reorientación de la democracia liberal en este final de siglo (R. Zapata, 2000a).

Teniendo como trasfondo este proceso, y con ánimo exploratorio, en las siguientes secciones defenderé que el incremento del desplazamiento físico de las personas a través de las fronteras estatales es una de las principales evidencias empíricas que causan precisamente esta necesidad de reconsiderar los vínculos entre el mercado, la inmigración y la justicia.

\section{Escenario 1: justicia, mercado, inmigración y pluralismo cultural}

La mayoría de los autores que examinan el pluralismo desde su dimensión cultural acostumbran a iniciar sus estudios con un preliminar analítico para 
distinguir sus ya aceptadas tres formas de expresión (B. Parekh, 1994; W. Kymlicka, 1995; F. Requejo, 1996). Mi posición al respecto es que se trata más bien de dos formas más una. Esta última, a pesar de expresar diversidad cultural, supone un estilo compartido de vida. Consecuentemente, prefiero designarlo como "pluralismo subcultural». Estoy hablando de aquellos grupos que optan por una forma de vida no-convencional (gays, lesbianas, movimientos sociales subculturales en general; I. M. Young, 1990). En contraste, las otras dos formas están relacionadas con grupos orientados por diferentes tradiciones culturales. Me refiero a la diversidad cultural expresada por la existencia de grupos territorialmente concentrados (las naciones sin Estado) y de grupos territorialmente dispersos (los inmigrantes).

Cada una de estas tres dimensiones de pluralismo cultural nos invita a abordar de forma diferente la relación entre la lógica del mercado y de la justicia. El pluralismo subcultural suele enfocarse hacia una demanda de participación política y de representación, una exigencia de inclusión y de reconocimiento dentro de la esfera pública; los grupos territorialmente concentrados expresan demandas de derechos culturales y reconocimiento diferencial con propuestas de arreglos institucionales en algunos casos, como un federalismo asimétrico (F. Requejo, 1995, ed. 1999).

En ambas formas se pide protección y derechos positivos, se enfatiza la necesidad de un ordenamiento justo frente a un dejar-hacer cultural que no da a todas las posibles manifestaciones de pluralismo cultural las mismas oportunidades de expresarse en la esfera pública.

Este artículo tratará de precisar la forma del pluralismo cultural que incluye fundamentalmente a los grupos de inmigrantes económicos extracomunitarios. $\mathrm{Al}$ respecto, existen al menos tres posiciones que tratan de resolver las tensiones originadas por la relación entre la inmigración y la justicia ${ }^{1}$.

Utilizando como indicadores la participación en la decisión y gestión política de la diferencia cultural, y el carácter cultural de la esfera pública, pueden precisarse las posiciones de cada orientación. Respectivamente, una exigencia de asimilación, de integración o de autonomía. Me interesa aquí adentrarme en los fundamentos que ayudan a legitimar el tratamiento estatal hacia los inmigrantes.

El modelo asimilacionista tiene como fundamento la defensa de un dejarhacer cultural (J. Gray, 1993). En él no se expresa ninguna necesidad de variar los límites y el contenido actual de los valores culturales que componen la esfera pública, eminentemente monocultural o cultural autóctona. Evidentemente, tampoco facilita la participación de los inmigrantes en las decisiones y gestión política de la diferencia cultural, en manos exclusivamente de los ciudadanos.

Las siguientes posiciones teóricas constituyen una de las bases de la investigación empírica que realicé con la ayuda de la Fundació Jaume Bofill, cuyo titulo final es: Esferas de justicia e inmigración: ¿de qué modo las instituciones públicas deberían acomodar a los inmigrantes? (marzo 2000). 
La coexistencia entre culturas diferentes es percibida como competencia cultural y, como tal, la cultura que dispone de más recursos para su supervivencia (la de los ciudadanos) podrá exigir a los otros las condiciones de acceso a una esfera pública controlada por ellos mismos. En los términos de los dos indicadores, la asimilación puede definirse como decisión y gestión política de la diferencia cultural en manos de la ciudadanía y carácter monocultural de la esfera pública. El inmigrante que quiera acceder a ella deberá, por lo tanto, dejar para la esfera privada aquellas prácticas culturales susceptibles de poner en tensión las ya establecidas. Este modelo es políticamente conservador en sus efectos, y sólo defiende la intervención estatal cuando la cultura autóctona es vista como amenazada por cualquier forma de «invasor cultural».

El modelo integracionista, el que tiene más adeptos, está orientado por ideas políticas liberales. Compartiría con la orientación anterior la defensa de que la decisión y gestión política de la diferencia cultural permanezca en manos de la ciudadanía, aunque admitiría algunas variaciones del contenido y de los límites culturales de la esfera pública para permitir que ciertas prácticas culturales de los inmigrantes tengan algún espacio. Este reconocimiento institucional tiene como impulso el deseo de apaciguar el conflicto cultural entre la población autóctona y la inmigrante, y obtener, así, un cierto grado de estabilidad social. En este caso, la esfera pública podría considerarse como pluricultural, aunque este contenido y sus límites estén todavía controlados por la ciudadanía. Las demandas de los inmigrantes que consigan pasar el «filtro» del control ciudadano podrán, así, ser públicamente practicadas y reconocidas (Ch. Taylor, 1992). La ciudadanía, no obstante, es quien en última instancia decide o no satisfacer dichas necesidades culturales de los extranjeros. En contraste con la orientación conservadora, la liberal rechazaría la asimilación cultural, pero exigiría a los extranjeros una integración. «Integración» porque, según el modelo, la ciudadanía estaría dispuesta a cambiar el carácter de su esfera pública, «interculturalizarla», si se me permite la expresión; pero sin posibilidad de participación política en la gestión de la diferencia cultural por parte de los extranjeros. En definitiva, las decisiones políticas todavía permanecerían exclusivamente en manos de los ciudadanos.

El modelo autonómico daría un paso más allá. Tendría una orientación política democrática. Compartiría con la liberal o integracionista la necesidad de crear una esfera pública sensible a las prácticas culturales de los inmigrantes. En tal caso, su exigencia sería de integración. Pero, en contraste con las dos anteriores orientaciones, estaría dispuesta a que los mismos inmigrantes residentes permanentes pudieran también formar parte del grupo decisor o, cuanto menos, como gestor de la diferencia cultural. En este sentido, por su carácter participativo, este modelo fomentaría la autonomía de los extranjeros. En una palabra, podrían participar en la toma de decisiones y en la gestión del contenido y de los límites de la esfera pública, como un ciudadano más.

Cualquiera que sea la posición que el Estado adopte, todos comparten la idea de que el problema de la coexistencia dentro de un Estado de inmigrantes 
y de ciudadanos tiene como centro una discusión sobre la justicia. Sigamos con el segundo escenario, que requiere introducir un lenguaje de justicia al fenómeno de la inmigración.

\section{Escenario 2: justicia, mercado, inmigración y Unión Europea}

En el proceso de construcción de la UE el problema descansa en el desarrollo asimétrico que estamos presenciando: el económico y el político. Cada uno tiene no solamente diferentes velocidades, sino sobre todo diferentes correspondientes motores: el Euro y la ciudadanía, respectivamente. La justicia a escala política es todavía un desideratum. El proceso económico tiene muchos años de ventaja (de experiencia y de respaldo institucional) en comparación con el joven proceso político. El acoplamiento todavía no se ha producido.

En esta situación existe una contradicción con consecuencias prácticas: existe un mercado único con su lógica de crear desigualdad, pero no su correspondiente contrapartida política, un ciudadano europeo, con derechos y recursos para hacer frente a los efectos negativos anteriores. La contradicción, el círculo vicioso práctico, está servido: los Estados nacionales todavía tienen, por ejemplo, competencias sobre el desempleo, mientras que su origen, el mercado, escapa de su control.

Además, cuando se aborda este tema desde la perspectiva de los extranjeros extracomunitarios (los euroextranjeros; R. Zapata, 1998b), la situación paradójica es mucho más evidente. Los euroextranjeros simplemente no tienen derecho a la tan preciada libertad de circulación dentro de las fronteras internas de la UE. Pueden tener derechos sociales cuando adquieren la posición de «residentes legales permanentes» ${ }^{2}$, pero, por el simple hecho de carecer de libertad de movimiento, no pueden disfrutar de esos derechos sociales en cualquier Estado de la Unión. Para los euroextranjeros, todavía existen muchas fronteras en la UE. La UE para ellos simplemente se está construyendo a sus espaldas (Zapata, 1999a).

Puede añadirse una nueva situación paradójica relacionada con la relación entre el mercado, la justicia y los extranjeros al nivel de la UE, como he tenido ocasión de señalar en otros trabajos (Zapata, 1998a). Si la UE desea realmente que el eurociudadano actúe como motor en el proceso de construcción política, es urgente iniciar un debate institucional sobre qué teoría de la inmigración debería limitarlo. Sin una política europea de inmigración, el eurociudadano está destinado a ser simplemente un puro instrumento en las manos de los

2 Como denizens, tal como comienzan a designarse a los «residentes permanentes» en el debate internacional. Estas personas se diferencian básicamente de los citizens porque carecen de derechos políticos, teniendo ya los otros derechos (civiles y sociales) prácticamente asegurados. Este término inglés, muy útil analíticamente, está falto de una traducción equivalente en nuestra lengua, de ahí que utilice su descripción. Que yo sepa el término fue introducido por T. Hammar (1989). 
diferentes Estados miembros. Si se quiere hablar de un eurociudadano como motor en el proceso de construcción de una eventual Unión política, debemos ser capaces de saber quién es euroextranjero, cosa que actualmente es imposible. Para poder hablar de una Unión política, debemos ser capaces de enfrentarnos a la difícil construcción de una política de inmigración única, y no de quince. En el momento en que se alcance este punto de discusión, deberemos también tener la precaución de orientarnos por principios de justicia. Sin esta predisposición, esta futura política europea de inmigración supondrá engrosar de nuevos elementos y justificaciones la ya compleja cuestión del déficit democrático.

Para iniciar una reflexión desde esta perspectiva nos debemos enfrentar a la situación asimétrica que viven los extranjeros europeos en los Estados miembros. Destacaría dos situaciones asimétricas básicas. La primera incide en que todavía hoy ser extranjero (por ejemplo, latinoamericano) y vivir en España no es lo mismo que serlo y vivir en Alemania. Los requisitos para un latinoamericano para alcanzar la ciudadanía europea vía artículo 8 del Tratado de la Unión Europea (accediendo a la nacionalidad y ciudadanía de un Estado miembro) no son los mismos en España que en Alemania, por poner dos ejemplos extremos. Existen, pues, y aquí quería llegar, diferentes vías para ser eurociudadano. El eurociudadano se ha convertido para el extranjero en una mercancía, absorbido por la esfera del mercado. Con un tratamiento desigual para llegar a ser eurociudadano, la justicia simplemente padece. La segunda situación se centra en la definición misma de la irregularidad. Existen prácticamente 15 situaciones diferentes para delimitar la irregularidad, dependiendo del Estado miembro. No existe todavía un criterio comunitario para definir la irregularidad. Para el euroextranjero, por lo tanto, el beneficio que supone entrar a formar parte de la población europea, como regular, es diferente según el Estado miembro. Para el euroextranjero no existe, pues, población europea, sino un club que le excluye de maneras diferentes. La justicia, en este segundo caso, también padece.

\section{Escenario 3: justicia, mercado, inmigración y el proceso de globalización}

Este tercer escenario tiene dos dimensiones. Hoy en día, los problemas de aplicación de políticas públicas deben abordarse de acuerdo con la doble lógica de la edad global que orienta la acción de los Estados (M. Albrow, 1996; R. Axtmann, 1996; Zapata, 1997): la lógica globalizadora económica, que tiene unas analogías evidentes con el viejo concepto de imperialismo y que hoy algunos autores denominan, por sus consecuencias, como McWorld culture (B. Barber, 1996); y la lógica globalizadora política o de los derechos humanos, de una humanidad común. Adoptar una posición adversa contra el proceso de mundialización económica es simplemente «darse de cabeza contra una pared», puesto que nadie puede negar su dinámica irreversible.

Al explorar la relación entre el mercado, la justicia y la inmigración en este marco debemos empezar por considerar dos hechos. Por un lado, y en compa- 
ración con la lógica globalizadora económica, la libertad de movimiento está sujeta a condiciones, la libertad de circulación de las personas no está en la misma línea que la del capital, de los bienes, de los servicios (B. Barry y R. Goodin, eds., 1992; J. F. Hollifield, 1992). Por otro lado, y comparándolo ahora con la lógica de los derechos humanos, la posición actual de los extranjeros es similar a la situación que prevalecía durante el siglo XIX (y gran parte del $\mathrm{XX}$ ), donde a la mayoría de la población se le negaba el derecho de pertenecer al pueblo (al demos), de votar, bajo criterios de exclusión basados en la propiedad y en el sexo (E. Balibar, 1992; J. de Lucas, 1994; D. Schnapper, 1994; J. Carens, 1989, 1995). Desde este prisma, el criterio que se usa para legitimar la exclusión ya no es ni el sexo ni la propiedad, sino la nacionalidad. Es, pues, lícito plantearse si el binomio nacionalidad/justicia puede en este nivel todavía funcionar (D. Miller, 1995; T. K. Oomen, 1997; D. Held, 1997; D. Zolo, 1997), y si estas dos nociones históricamente conectadas son simplemente compatibles desde el punto de vista de una política de inmigración. Algo debe hacerse: las políticas de inmigración basadas en el criterio de nacionalidad contradicen la realidad económica y política de esta new global age.

Conectando estas dos lógicas, puede decirse, como en el escenario anterior, que existe asimetría. Si se considera que tras la globalización económica está el mercado sin justicia, la lógica política globalizadora debería estar orientada por principios de justicia. Su objetivo debería ser contrarrestar los efectos de la anterior, del mercado global. Sin esta función de control, se le deja abierta la puerta a la globalización económica para que vulnere los derechos humanos más elementales, sobre todo (aunque no únicamente) en el Tercer Mundo. Desde la perspectiva de la inmigración, este problema incide en que los inmigrantes están simplemente desnudos ante el mercado global, no poseen instrumentos para frenar sus efectos sobre sus vidas. Idealmente, una justicia global debería prevenir los efectos negativos del mercado global; en la práctica, todavía hay mucho camino que recorrer (Zapata, 1999c).

Estos tres escenarios justifican la necesidad de introducir un lenguaje de justicia con el objetivo de regular las contradicciones que hemos señalado. En la siguiente parte exploraré esta vía de análisis en el contexto del Estado.

\section{EL LENGUAJE DE LA JUSTICIA EN LA RELACIÓN ENTRE EL MERCADO Y LA EXTRANJERÍA}

M. Walzer es uno de los primeros teóricos que detecta problemas prácticos del capital conceptual del liberalismo para incorporar el fenómeno de la inmigración en una teoría de la justicia. Nos lega la necesidad de reconceptuar algunos aspectos de la teoría liberal con el fin de reorientar su práctica estatal. Avanzando algunos argumentos defendidos hoy en día por W. Kymlicka (1989a, 1995), el principal problema práctico de la teoría liberal de la justicia es que ha supuesto una homogeneidad cultural de la comunidades políticas 
sobre las que teorizaba ${ }^{3}$. Este implícito liberal plantea la relación entre la cultura, el mercado y la política, y de alguna forma incide en la necesidad de separar estas esferas, tal como antes ocurrió con la esfera de la religión.

El mensaje básico de Walzer es la necesidad de reconsiderar la diferencia cultural como un bien colectivo que debe ser incorporado en la lista de los bienes primarios liberales. Éste es uno de los esfuerzos que destina en uno de los primeros capítulos de Spheres of Justice (1983, SJ). Una de las cuestiones que plantea el pluralismo cultural a la teoría liberal de la justicia es si es justo que los Estados hagan funcionar sus economías con personas excluidas de la ciudadanía $(S J, 55)$. Es decir, si los Estados pueden continuar excluyendo de la esfera de las decisiones a personas por el simple hecho de su nacionalidad ( $S J$, 54). La naturaleza convencional (sin legitimación religiosa o natural) del acceso al territorio indica que es un bien primario regulado, como todo bien distribuido por el Estado, a través de determinados criterios. Para Walzer, y aquí reside la fuerza de su argumento, los criterios de acceso que la tradición liberal utiliza para legitimar su práctica son de dudosa justiciat.

El criterio del territorio plantea, por ejemplo, el problema de que es considerado como un bien social $(S J, 44-45)$ y, como tal, como un espacio protegido de vida (a protected living space). La cuestión básica que suscita es si una comunidad política puede excluir a los necesitados simplemente porque son extranjeros. La tradición liberal demuestra que el principio de la ayuda mutua (principle of mutual aid) ha sido practicado. Ahora bien, este principio ha sido implícitamente aplicado con la debida precaución utilitarista de que el número de extranjeros admitidos no empeorará la cantidad de libertad que los miembros de hecho tienen en su territorio. La admisión, una vez que el principio de ayuda mutua es inaplicable por causa del número, está orientada implícitamente por la afinidad ideológica y, por lo tanto, sigue siendo en la práctica discriminatoria (SJ, 49-50). Asimismo, existen criterios demográficos, como el que enfatiza el suficiente espacio físico y la densidad de la población para permitir a otros el beneficio del bienestar. Estos criterios basados en la riqueza y los recursos disponibles están también regulados por principios utilitaristas. Dan por supuesto que es lógico que se limite la inmigración tan pronto como ésta pueda interferir materialmente con los esfuerzos del gobierno para mantener un alto nivel de vida para sus ciudadanos, especialmente las clases autóctonas más desposeídas $(S J, 48)$.

3 En una trabajo reciente Walzer enfatiza que evidentemente la homogeneidad cultural de la sociedad nunca ha existido. Cuando se habla en estos términos nos referimos a que "a single dominant group organises the common life in a way that reflects its own history and culture and, if things go as intended, carries the history forward and sustains the culture. [...] Among histories and cultures, the nation-state is not neutral; its political apparatus is an engine for national reproduction» (Walzer, 1997; 25). En última instancia, lo que justifica la práctica del grupo cultural dominante es una pasión humana por la supervivencia a través del tiempo, llegando a legislar como si una única tradición existiera: la suya propia.

${ }^{4}$ No es mi objetivo presentar todos los criterios que avanza Walzer, para ello remito a mi próximo libro R. Zapata (2000a). Véase también R. Zapata (1999b). Aquí sólo introduciré los que sean pertinentes para mi enfoque. 
En resumen, para Walzer, el principio de justicia debe ser el siguiente: que el proceso de autodeterminación soberana en el cual un Estado configura su vida interna debe ser igualmente abierto para todos. Este tema se ha convertido incluso en problema estructural: sin la negación de los derechos políticos y la permanente amenaza de la exclusión de la población inmigrante, el sistema simplemente no funcionaría. Este supuesto consentimiento liberal no es legítimo para cualquier teoría de la justicia $(S J, 58)^{5}$.

Un esfuerzo por analizar la extranjería desde el punto de vista de la justicia es, pues, necesario. Esta línea de investigación se está abriendo desde la segunda mitad de los años noventa. Esta preocupación sigue una lógica derivada de la reflexión de acomodar la ciudadanía en los cambios de parámetros de nuestras sociedades occidentales (por ejemplo, F. Requejo, 1998a, b). Entre la literatura que ya ha comenzado a aparecer existe una convicción generalizada de que la relación entre la justicia y la extranjería debe explorarse siguiendo dos criterios legitimadores: la nacionalidad y el mercado. Cada uno de ellos está al origen de una concepción del extranjero: el extranjero como un vehículo de valores culturales que entran en conflicto con los de la nacionalidad de recepción, y el extranjero considerado como una mercancía para la comunidad de recepción. Ya he tenido ocasión de ocuparme del primer criterio legitimador, el de la nacionalidad (R. Zapata, 2000b). Mi objetivo ahora es explorar el contenido del segundo, a saber, el económico.

Actualmente, el principio que guía las prácticas del Estado liberal es el mercado en general, la situación del mercado laboral nacional en particular. En teoría, el liberalismo ha defendido la libertad de movimiento de las personas al mismo nivel que el del capital (J. Carens, 1987, 1997; J. Hollifield, 1992). En la práctica, el criterio para admitir a los extranjeros plantea problemas de justicia, puesto que las personas son tratadas de forma desigual en función de criterios estrictamente utilitaristas. Se parte del convencimiento de que es normal que los Estados tengan derecho de adoptar cualquier práctica hacia la extranjería siempre que sea beneficioso económicamente.

Desde esta perspectiva generalizada, si la inmigración es susceptible de incrementar el desempleo o la política fiscal asociada con programas sociales, los Estados son libres de decidir limitar el acceso. En contraste, si la inmigración demuestra ser ventajosa económicamente (recordemos, por ejemplo, las políticas de los «trabajadores invitados»), entonces se suele tomar generalmente como una razón suficiente para aceptar más inmigrantes. Aquí se percibe con claridad la percepción del inmigrante como una mercancía. Ante estas constata-

Es cierto que Walzer proporciona también argumentos a favor de la libertad de elección de los extranjeros al nivel de acceso. Piensa que una vez los extranjeros han sido admitidos deben ser tratados como cualquier ciudadano, pero no pondera suficientemente que existe una obligación para admitirlos (agradezco a I. M. Young estas precisiones). No obstante, su lógica nos permite aceptar que a pesar de defender límites en la admisión, éstos deben ser iguales (ésta es la palabra clave en este nivel) para todos los extranjeros, independientemente de su nivel económico o su formación técnica. 
ciones iniciales, las preguntas que me orientarán son las siguientes: ¿es justo la selección de inmigrantes en base a lo que pueden contribuir económicamente?, ¿es justo dejar a las organizaciones empresariales en su función de grupo de presión más bien que a los gobiernos decidir qué grupos de inmigrantes son susceptibles de contribuir a la producción nacional?, ¡es justa la política de cuotas favorecida por los Estados liberales para regular el acceso de los extranjeros?

El punto de partida de este lenguaje de la justicia es que todo extranjero tiene el derecho de ser plenamente tratado como igual. Todo Estado que restrinja esta posibilidad actúa contra el principio básico de la igualdad. El primer argumento que expresa este lenguaje es que para que una ley de extranjería sea justa debe permitir que los inmigrantes puedan ejercer su derecho de ser tratados iguales independientemente de sus propiedades económicas (formación, renta, etc.) y de la situación nacional del mercado laboral. Los extranjeros son ante todo personas y, como tales, su derecho a tener igual respeto (entendido kantianamente como el derecho a ser tratado como un fin y no como un recurso; Ch. Larmore, 1990) debe garantizarse.

Tal como sugiere este breve recorrido teórico, el lenguaje de la justicia respecto a la inmigración está lejos de ser simple. La relación entre la justicia y la extranjería refleja en última instancia las cuestiones fundamentales de los límites de las políticas estatales. ¿Hasta qué punto es legítimo para los Estados liberales dejarles perseguir sus intereses económicos, y hasta qué punto se les puede pedir que se preocupen también de los intereses de los extranjeros? ¿Está todo permitido o existen límites a las prácticas estatales? Si existen, ¿cuáles son? Y una vez determinados, ¡cómo institucionalizarlos? Mi contribución en este artículo es explorar la posibilidad de ofrecer algunas respuestas a estas últimas preguntas. Tomaré la Ley de Extranjería y su Regulación de 1996 como estudio de caso ${ }^{6}$.

\section{MERCADO, JUSTICIA E INMIGRANTES EN LA LEY DE EXTRANJERÍA ESPAÑOLA}

Mi argumento es que la Ley de Extranjería del Estado español vulnera el principio de justicia básico de la igualdad. Limitaré mi evaluación al nivel de acceso, dejando de lado por el momento otro nivel de discusión no menos interesante, el de la coexistencia entre inmigrantes y ciudadanos en un mismo espacio territorial, una vez que han sido admitidos.

El primer contacto que tienen los extranjeros cuando entran en un Estado es con el mercado. Ésta ha sido la primera idea de integración que ha orientado cualquier política de inmigración en Europa: los extranjeros han sido perci-

${ }^{6}$ Dadas las circunstancias en su elaboración y rápida aprobación de la nueva Ley 4/2000, sin tener todavía una regulación y posiblemente su futura modificación, creo preferible centrarme en la Ley de extranjería de 1985. En cualquier caso, los argumentos que defenderé a continuación todavía son válidos con la actual Ley 4/2000, y por lo tanto, su aparición de ningún modo los invalida, sino más bien los refuerza. 
bidos antes como trabajadores que como personas (Z. Layton Henry, ed., 1990). Mi intención es examinar desde el punto de vista de la justicia los efectos que tiene dicha percepción en la Ley española. Mi premisa es que cuando el mercado es considerado como prioritario y condicionante para la regularización del estatus de los extranjeros estamos enfrentados con prácticas de dudosa justicia. Este problema adopta la forma siguiente: la mayoría de los extranjeros que no son, o no pueden ser, «mercantilizados» (commodified) son categóricamente rechazados o, si fueron previamente admitidos, expulsados. Desde esta premisa, examinaré el siguiente argumento: una politica de inmigración tiene un carácter más justo cuando el mercado no funciona como criterio de orientación.

En la primera sección introduzco el significado de justicia y de extranjero que considero adecuado para evaluar la Ley de extranjería española. En la segunda sección ilustraré mi argumento destacando los límites de dicha Ley. Al tratar este tema abordaré dos situaciones donde el mercado es utilizado como criterio de discriminación: el de adquirir residencia permanente y el de adquirir derechos económicos. En ambos casos, el objetivo de mi análisis es detectar cuándo la extranjería es tratada como una mercancía.

\section{Definiendo "justicia» $y$ "extranjero»}

Significaré por «justicia» el resultado de satisfacer el principio de igualdad, el cual depende de su correspondiente fundamento de inclusión. Puede definirse en términos negativos como el principio de no-discriminación ${ }^{7}$. Este principio incide en que la igualdad puede ser percibida bajo dos formas: igualdad de tratamiento e igualdad de oportunidades. La primera insiste en que un tratamiento diferenciado entre nacionalidades de origen por razones económicas no es justo, y está conectado con la legalidad de una política de inmigración; la segunda, que una diferenciación de condiciones no es justo, y está vinculado con el mínimo de bienestar que una política de inmigración tiende a garantizar.

$\mathrm{Mi}$ argumento es que este principio juega un papel fundamental cuando se aplica en el nivel de acceso y depende del fundamento de la inclusión. En efecto, al introducir el elemento de la extranjería dentro de una reflexión amplia sobre la justicia, uno de los principales problemas con los que nos encontramos es el de caracterizar los criterios de inclusión y de exclusión dentro del territorio (Walzer, 1993). La inclusión aparece, pues, como uno de los fundamentos distintivos del principio de la igualdad.

La exclusión tendría, a su vez, dos usos. Puede aplicarse a todos los extranjeros, sin discriminación entre nacionalidades y personas por razones de mercado, o puede depender de variables como su formación técnica, su nivel eco-

Entiendo la «discriminación» como siguiendo una lógica de jerarquización que implícitamente supone una relación de dominación (M. Wieviorka, 1998; 64). 
nómico, sus rentas, etc. Es decir, la exclusión puede ser universal o selectiva: puede discriminar entre extranjeros y ciudadanos o entre los mismos extranjeros, respectivamente. En términos del principio de igualdad, la exclusión selectiva tiene un menor grado de justicia que la exclusión universal, puesto que legitima la selección sobre la base de los recursos económicos y de la formación de los extranjeros y/o sobre la base de las «necesidades» del mercado laboral estatal. En este caso cobra sentido mi argumento de que el extranjero es considerado como una mercancía.

Por «extranjero" entiendo un concepto dependiente del eEstado, a saber, las condiciones y normas que regulan sus actividades dependen de la soberanía estatal, el único componente activo de la relación vertical ${ }^{8}$.

Teniendo en cuenta estas consideraciones previas, mi propósito es utilizar este principio de igualdad (de no-discriminación selectiva) como instrumento analítico para evaluar los límites de la Ley de Extranjería española.

\section{Los limites de la Ley de Extranjería española9}

Se sabe que la inmigración es un problema emergente en España, históricamente un país de emigración. Desde la segunda mitad de los años ochenta se ha producido este cambio de dirección ${ }^{10}$. La primera regulación del estatus de este nuevo flujo de inmigrantes se produjo en 1985, como imperativo, como se sabe, de las negociaciones para incorporarse a la entonces llamada Comunidad Europea (1986). Ésta es la llamada Ley Orgánica 7/1985, de 1 de julio, sobre Derechos y Libertades de los Extranjeros en España (de aquí en adelante, como LOE). La última versión extensiva de su Regulación es de 1996 (Real Decreto 155/1996, de 2 de mayo, Rglto), que introdujo, como uno de los cambios significativos en cómo la $L O E$ debe implementarse, un reconocimiento institucional de la naturaleza permanente de la inmigración.

${ }^{8}$ Es obvio que el poder estatal para determinar estas condiciones no es ilimitado, sino que depende del marco Internacional y de la Unión Europea. El primero tiene dos caras: las normas del Derecho internacional en general; y en particular, los acuerdos y tratados bilaterales o multilaterales que dicho estado ha ratificado (A. Cassese, 1993). El segundo, depende del marco de la UE, quién cada vez tiene más competencias en materias de acceso, sobretodo a partir del Tratado de Amsterdam de 1998 (R. Zapata, 1999a, 2000b). El análisis que sigo distingo claramente entre estos "deberes internacionales y comunitarios del eEstado" y la práctica estatal en una ley de extranjería (V. Estrada, 1989; 32).

${ }^{9}$ Véase nota 6.

${ }^{10}$ La literatura demográfica y sociológica española de los noventa comienza a detectar el cambio que se está produciendo en España. En efecto, a pesar de ser un país que continúa teniendo más emigrantes que inmigrantes, la tendencia es hacia un cambio de dirección. En 1991, había 1.624.211 de residentes españoles fuera de nuestras fronteras (contra 2008026 en 1980), y 407671 residentes extranjeros en nuestro territorio en 1990 (contra 182045 en 1980. En 1996 el número se ha incrementado a 538.984. Véanse, entre otros, M. A. de Prada (1989), A. Izquierdo (1992, 1996), R. Aragón Bombín y J. Chozas Pedrero (1993; 20-1). 
Asimismo, España ha firmado los más importantes Tratados Internacionales, los cuales juegan un papel regulador en su Constitución. Estos límites mínimos de la actividad de los extranjeros se mencionan explícita o implícitamente en el artículo 10 de la CE (Constitución Española) ${ }^{11}$. Gracias a estos Tratados, se satisface formalmente el principio de la igualdad. Desde la perspectiva que he trazado, estos mínimos coinciden con los límites mínimos de la justicia ${ }^{12}$. A pesar de ello, cuando se observan las condiciones específicas de la Ley de Extranjería se constata que este principio no se satisface plenamente. Esto puede justificarse examinado la posición constitucional de los extranjeros.

Los límites constitucionales de la actividad de los extranjeros aparecen en el Título I de la CE. El principio de igualdad es incorporado en virtud de las restricciones internacionales, pero también el principio de discriminación. En efecto, estos límites mínimos están vinculados a la dignidad de la persona, en concordancia, como ya he avanzado, con los Tratados Internacionales ${ }^{13}$. Más allá de estos límites opera el principio universal de la exclusión, esto es, una serie de derechos y de libertades constitucionales literalmente referidos a los españoles y a los nacionales. El primero de ellos es significativamente el derecho a la igualdad ante la ley (art. 14) ${ }^{14}$, que legitima constitucionalmente la desigualdad de trato entre españoles y extranjeros.

Para ilustrar el argumento que quiero defender, me concentraré en los criterios para acceder a la población, los de admisión. Al tratar este nivel podemos distinguir dos posibilidades: la de la residencia y la del trabajo. Examinaré cada una de ellas por separado.

${ }^{11}$ Explícitamente, la Declaración Universal de los Derechos Humanos (1948), e implícitamente, la Convención Europea de Protección de los Derechos Humanos y de las Libertades fundamentales (1950, ratificado en 1979, 26 septiembre), el Convenio Internacional de los Derechos Civiles y Politicos y el Convenio Internacional de los Derechos económicos, sociales y culturales (1966, ratificado en 1977, 13 abril).

${ }_{12}$ Sus pilares básicos son, recuerdo: protección de la vida e interés frente a la violencia, no detención arbitraria, no ser torturado ni sometido a tratamientos inhumanos, tutela judicial, ejercicio de derechos básicos, entre los que se incluyen los admitidos como derecho de familia (R. Ma Ferrer Peña, 1989; 28).

${ }^{13}$ Los principales derechos y libertades que el extranjero tiene como persona, son, evidentemente, el derecho a la vida e integridad física y moral (art. 15) y, por extensión, protección a la salud (art. 43) y protección a la familia y a la infancia (art. 39); derecho a la libertad y a la seguridad personales, esto es, a la detención legal o Habeas Corpus (art. 17), y por extensión, derechos procesales o a la protección judicial de los derechos (art. 24) y a la legalidad sancionadora (art. 25); al medio ambiente (art. 45); así, como las imprescindibles libertades de creencias tanto religiosas como ideológicas (art. 16), y, por extensión, derecho a la intimidad (art. 18) y a la libertad de expresión (art. 20), derechos de reunión y de manifestación (art. 21) y de asociación (art. 22), y derecho a la cultura (art. 44).

${ }^{14}$ El artículo 14 dice: «Los españoles son iguales ante la ley, sin que pueda prevalecer discriminación alguna por razón de nacimiento, raza, sexo, religión, opinión o cualquier otra condición o circunstancia personal o social» (énfasis añadido). 


\subsection{Trabajadores extranjeros y el estatus de la residencia permanente}

La residencia da derecho al extranjero a pertenecer a la población española, aunque no a su demos. Interesa analizar los requisitos para poder ser miembro de la población española.

Antes de comenzar es necesario presentar la tipología que permite establecer la legislación. Para mis propósitos, puede reducirse a dos: el tipo de residencia no-permanente (que incluye la inicial y la ordinaria) y la permanente ${ }^{15}$.

Argumentaré que el criterio para distinguir estos dos tipos de residencia vulnera directamente el principio de justicia. Si nos concentramos en las variables que enmarcan el tipo permanente pueden destacarse cuatro criterios: la independencia económica respecto al mercado de trabajo nacional (jubilados y pensionistas extranjeros), la ius soli (haber nacido en territorio español), la que establece un vínculo de parentesco con personas de nacionalidad española (matrimonio, ascendientes o descendientes que sean originariamente españoles) y, por último, la afinidad histórica, geográfica y cultural (iberoamericanos, portugueses, filipinos, andorranos, ecuatoguineanos, gibraltareños y sefardíes). En resumen, este tipo permanente de adquisición del permiso de residencia excluye a todos aquellos que sean económicamente dependientes y que no tengan ningún vínculo con España, o bien a través del territorio (nacimiento), o bien a través de su relación de parentesco con la nacionalidad española (cónyuges, ascendientes, descendientes), o bien a través de la relación cultural y política de sus respectivos países con la nación española.

El mercado es, pues, uno de los criterios que excluye y discrimina selectivamente. Desde este prisma puede extraerse al menos una conclusión: la relación de prioridad que tienen los tres últimos criterios respecto al mercado. Es decir, pueden optar a la residencia permanente todos aquellos extranjeros que cumplan los tres últimos criterios independientemente de si son o no económicamente dependientes del mercado laboral; o, haciendo una lectura inversa, sólo pueden optar a la residencia permanente aunque no cumplan los tres últimos criterios aquellos que sean económicamente independientes del mercado laboral. Por lo tanto, el mercado no afecta directamente a aquellos que entran dentro de las otras tres situaciones. El mercado se utiliza, pues, como criterio a posteriori y no a priori para determinar el tipo permanente, en contraste con los del tipo nopermanente (inicial u ordinario), donde el mercado actúa como criterio determinante a priori. Todos aquellos extranjeros (su mayoría) que no cumplen uno de los tres últimos criterios deberán pedir el estatus de residencia no permanente.

15 Estos tipos de residencia mantienen una relación. En la base está el tipo inicial (validez máx. un año, y tras renovación, dos años más), que como su nombre indica es el primer estatuto legal que tendrá el extranjero. Este tipo inicial opera como condición para poder acceder al tipo ordinario (validez máx. tres años) y también puede actuar como condición, aunque no necesariamente, para poder optar por el tipo permanente (arts. 49-55 Rglto). El problema estriba en que uno puede tener acceso al tipo permanente habiendo tenido previamente el primero. Depende de su origen nacional y, lo que nos interesa aquí enfatizar, de un criterio económico. 
Una vez analizadas las principales condiciones que se requieren para poder pertenecer a la población, y ponderado que el criterio del mercado actúa como uno de los principales referentes para la mayoría de las personas que desean instalarse en España, vulnerando, de este modo, el principio de justicia de la igualdad, me adentraré en las restricciones que tiene el extranjero para poder optar a los derechos económicos. Examinaré directamente la relación del extranjero con el mercado nacional, teniendo en mente la conclusión anterior.

\subsection{Trabajadores extranjeros y el mercado laboral nacional}

Según la CE, el derecho al trabajo es exclusivo de los españoles (art. 35). Esto no implica que los extranjeros no puedan constitucionalmente tener acceso al mercado laboral nacional, sino que sus relaciones con el mercado están condicionadas por una ley que les discrimina universalmente frente a los nacionales. Por lo tanto, según la CE, el principio de igualdad queda supeditado a unas bases jurídicas especiales ${ }^{16}$.

Aunque sea altamente conocido, déjenme empezar recordando que existe una relación de implicación entre permiso de trabajo y de residencia. Esto supone que la posición de residente no implica necesariamente el derecho al trabajo, pero no lo contrario. Además, el apartado 3 del artículo 15 de la $L O E$ insiste en que ambos permisos deberán ser de «idéntica duración [máximo 5 años] y se expedirán en un documento unificado, cuya obtención, y en caso de renovación, se ajustará, asimismo, a un procedimiento único que se determinará reglamentariamente».

Esta relación no sería un problema de justicia si no se discriminara selectivamente, como efectivamente ocurre. En efecto, la relación de implicación entre permiso de trabajo y de residencia sólo afecta a los extranjeros no comunitarios. Se confirma de este modo mi argumento de que si bien se formaliza un principio de igualdad de trato, desde el punto de vista de las exigencias requeridas se sigue el principio de exclusión selectiva entre tres tipos de personas: los nacionales, los comunitarios y los no comunitarios.

La legislación española diferencia dos categorías de trabajadores (art. 75 Rglto): los trabajadores por cuenta ajena y por cuenta propia. Dentro de estas dos categorías se contemplan también subcategorías, cuyo criterio diferenciador ya no es la dependencia o independencia a un empresario, sino la temporalidad, el tiempo de trabajo. Esquemáticamente:

16 Tendré básicamente en cuenta el Título III de la $L O E$ (arts. 15-18) y el cap. IV de su Rglto (arts. 68-96). Para más detalles véase, entre otros, a P. Biglino Campos (coord.) (1998). A pesar de las fechas, continúan siendo interesantes J. Cardona (1985, 1989), M. Ramos (1989) y M. Polo (1994). 
Trabajadores Tipo A: 9 meses por cuenta Tipo B: 12 meses ajena
1. Esfera de actividad: no puede cambiar de residencia y de trabajo durante el período de permiso concedido, bajo amenaza de expulsión.

2. Criterio: protección de la fuerza nacional laboral: los extranjeros que soliciten trabajo lo obtendrán, si y sólo si, ninguna persona nacional ha solicitado el mismo trabajo.

3. Circunstancias de denegación:

3.1. "cuando lo aconseje la situación nacional de empleo";

3.2. "cuando concurra cualquier otra causa que sea considerada motivo legítimo por la autoridad laboral, mediante resolución debidamente motivada».

Tipo C: 3 años Permite movilidad geográfica y laboral.

Trabajadores Tipo D: 12 meses por cuenta Tipo E: 3 años propia
Sin límites. Renovación «si subsisten las mismas o análogas circunstancias que determinaron la primera concesión».

Dentro de la primera categoría, la de cuenta ajena, existen tres subcategorías dependiendo de si la duración es como máximo de nueve meses (permiso A), doce meses (permiso B) o tres años (permiso C). En la segunda categoría, la de cuenta propia, existen dos subcategorías, la de duración máxima de doce meses (permiso D) y de tres años (permiso E). Teniendo estas categorías y subcategorías como base, podemos comenzar a analizar las variables que entran en juego en cada caso susceptibles de ofrecernos información para evaluar su grado de justicia.

Respecto a los tipos A y B, los de corta duración, interesa destacar que están supeditados a unas circunstancias limitadas como son la de domicilio y contrato de trabajo. El extranjero no puede simplemente cambiar ni de domicilio ni de puesto de trabajo durante el permiso concedido, so pena de expulsión. Se constata así, y de nuevo, que el principio de igualdad de condiciones queda vulnerado, puesto que los trabajadores extranjeros de los tipos A o B no tendrán iguales oportunidades que los otros para poder exigir condiciones laborales equiparables a los nacionales: pesará más el despido o la no renovación del contrato, con las consecuencias negativas que implica.

Igualmente, en la lista de requisitos para la concesión o denegación de estos permisos de corta duración (A y B), el Rglto manifiesta explícitamente medidas concretas de protección para la mano de obra nacional, puesto que exige al empresario justificación de publicidad del puesto de trabajo, dejando 
de este modo por implícito que el extranjero demandante sólo podrá optar al trabajo si ningún nacional ha expresado su deseo de ocuparlo. Pero más contundentes al respecto son las circunstancias previstas para su denegación (art. 82 Rglto). Para mis propósitos destacaré dos de ellas. La primera justifica la denegación "cuando lo aconseje la situación nacional de empleo», y la segunda deja formalmente abierta la actuación discrecional de la Administración en tanto que establece que la denegación podrá ser justificada «cuando concurra cualquier otra causa que sea considerada motivo legítimo por la autoridad laboral, mediante resolución debidamente motivada». De nuevo aquí se vulnera el principio de igualdad por razón de interés del mercado laboral nacional, es decir, discriminación selectiva. En contraste con estas dos primeras subcategorías (A y B) claramente excluyentes, el tipo $\mathrm{C}$ facilita la movilidad laboral, tanto desde el punto de vista geográfico como sectorial (art. 75.4 Rglto).

Es también relevante evaluar la cuestión relativa a las circunstancias que legitiman la concesión y la renovación del permiso de trabajo (art. 18 LOE). Los principios de discriminación y de exclusión rigen con claridad los dos primeros apartados, en tanto que se formaliza como legítimo para la denegación "la existencia de trabajadores españoles en paro en la actividad que se proponga desempeñar el solicitante» y "la insuficiencia o escasez de mano de obra española en la actividad o profesión y zona geográfica en que se pretenda trabajar». Aquí de nuevo la soberanía del mercado laboral nacional opera como razón suficiente para condicionar el acceso legal al mercado laboral para el extranjero. El mercado en este caso opera de nuevo como variable determinante para adoptar principios contrarios a la justicia.

Tal como ocurría para la concesión de la residencia, existe una lista explícita de preferencias o de trato desigual sobre la base de unos criterios determinados. Es difícil hallar un hilo conductor coherente que justifique esta lista (arts. 77, 79-80 Rglto). Estos criterios están vinculados directamente con el territorio nacional (ius soli, residentes durante los últimos dos años), una determinada relación de parentesco con los nacionales (matrimonio, ascendientes o descendientes nacionales, parentesco de primer grado con empresario que le contrate, ser cónyuge o hijo de extranjero que ya tenga permiso de trabajo), nacionales de origen o descendientes de personas originariamente españoles, y las afinidades culturales e históricas de España (iberoamericanos, portugueses, filipinos, andorranos, ecuatoguineanos, sefardíes y gibraltareños), además de los cuerpos técnicos y de confianza.

En conclusión, y respecto a estos claros ejemplos de discriminación selectiva formalizada ${ }^{17}$, cabe mencionar que la legislación también restringe su uso

${ }^{17}$ La $L O E$ insiste en este principio de discriminación en tanto que lo formaliza explícitamente en el artículo 23 dentro del Título $\mathrm{V}$ dedicado a los regímenes especiales. Vale la pena transcribir literalmente el articulado: «los nacionales iberoamericanos, portugueses, filipinos, andorranos, ecuatoguineanos, sefardíes y las personas originarias de la ciudad de Gibraltar cuando pretendan realizar una actividad lucrativa, laboral o profesional, por cuenta propia o ajena, tendrán preferencia para trabajar en España, sobre otros extranjeros conforme se establece en el 
proporcionando a la Administración la facultad de ejercer discrecionalidad para modificar las preferencias establecidas sobre la base de dos criterios: la situación nacional de empleo y el trato de reciprocidad que reciban los trabajadores españoles. En cualquiera de los dos casos, el ejercicio de la soberanía española respecto al control del mercado nacional laboral es el último argumento. Por lo tanto, la discriminación y exclusión selectivas son los principios que regulan la relación entre los extranjeros y el mercado laboral español.

\section{IV. ÚLTIMAS OBSERVACIONES: LA INMIGRACIÓN ES UN PROBLEMA ESTRUCTURAL}

Este artículo exploratorio ha defendido el argumento siguiente: una ley de extranjería tiene un carácter más justo cuando el mercado no funciona como criterio de orientación para discriminar entre las diferentes nacionalidades demandantes de acceso a nuestra población. En el caso de la legislación española se ha constatado que el mercado funciona como criterio para legitimar principios de discriminación y de exclusión selectivas. Este hecho no es exclusivo de España, sino que pertenece a la propia configuración de la mayoría de las leyes de extranjería. En el caso de España, esta conclusión general podría adoptar la siguiente forma: descartar toda exclusión y discriminación selectivas, es decir, no diferenciar en las circunstancias económicas y de formación técnica de los extranjeros que deseen acceder en nuestro país al aplicar la igualdad de tratamiento y de oportunidades. Esto implica reducir hasta un mínimo toda presencia de discrecionalidad administrativa al respecto, es decir, las justificaciones para la exclusión y la discriminación entre los diferentes demandantes basadas en la soberanía del Estado respecto al mercado laboral nacional. En este nuevo escenario desaparecerían gran parte de los elementos que permiten todavía hoy considerarlo como una mercancía y un recurso regido simplemente por principios utilitaristas.

El mensaje principal que he querido transmitir es que el inmigrante no es una mercancía, ni la inmigración debe ser considerada como una carga o un beneficio para la sociedad receptora. Esta visión instrumental del fenómeno tiene quizás la virtud de la simplicidad, pero políticamente debería ser tema de debate. La inmigración es más bien un problema estructural. Los políticos deberían tomar consciencia de ello. Esto implica que deberían concentrarse en analizar cómo afecta la llegada de inmigrantes a nuestras propias estructuras institucionales, y estudiar vías para su modificación para poder incluirlos como

artículo 18.3 y no vendrán obligados al pago de las tasas correspondientes por la expedición de permisos de trabajo». Cabe insistir que el principio de discriminación opera respecto a los otros extranjeros y no respecto a los nacionales españoles. Haciendo una lectura teniendo como referencia a estos últimos, el principio de justicia que expresa este artículo es claramente el de la equiparación, es decir, el de la igualdad y el de la inclusión. 
nuevos receptores. En lugar de ello, recurren a las lógicas utilitaristas que históricamente ha caracterizado el tratamiento estatal de la inmigración.

$\mathrm{Al}$ abordar el tema de la inmigración, nuestros políticos deben comenzar por reconocer que nuestros Estados encuentran serias dificultades para gestionar las demandas de los inmigrantes porque tradicionalmente su estructura institucional fue construida sin tener en cuenta la multiculturalidad, esto es, la posibilidad de coexistencia de culturas y tradiciones diferentes.

La historia europea sobre la inmigración muestra esta dificultad. En lugar de plantear seriamente la cuestión, de repensar los pilares básicos estructurales de nuestras sociedades, y de aceptar que deben modificarse algunos de sus aspectos para integrar a los inmigrantes, han recurrido a una lógica instrumental, sin ninguna voluntad política reestructuradora.

Veamos brevemente cómo se ha utilizado esta lógica históricamente y cuáles fueron sus efectos. Tras la Segunda Guerra Mundial se abrió el proceso de reconstrucción económica de Europa. Para lograrlo, se vinculó explícitamente el desarrollo económico y la demanda de mano de obra de terceros países. Aparecieron incluso programas explícitos para fomentar la inmigración. Esta prioridad utilitarista dejó en segundo plano aspectos regulativos de la población que llegaba, sin planificación sectorial, geográfica, comunitaria. Se llegaron a establecer acuerdos bilaterales con los países emisores de «mercancías humanas» (Gran Bretaña, Alemania y Francia con Italia, Portugal, España, Turquía, Argelia, India, por ejemplo). La demanda era básicamente estructural, pero se pensaba que los inmigrantes regresarían a sus países. A esta etapa de Puertas Abiertas (1945-1973) le siguió una etapa de Puertas Cerradas (1973-1990). Esta segunda fase se inició con la crisis económica de los Estados de Bienestar en los años setenta. Se vinculó dicha crisis con la constatación de que la mayoría de los inmigrantes se quedaba. El «problema de la inmigración» comenzó a aparecer. Su contenido era, de nuevo, utilitarista: la inmigración suponía unos costes sociales y políticos mayores que los económicos. La OCDE llegó incluso a pronunciarse al respecto. Comenzaron a aparecer los debates sobre los «límites de la tolerancia» (principalmente en Francia), o hasta qué punto un país puede soportar la llegada de inmigrantes sin que suponga una disminución de la calidad de vida de sus ciudadanos, de sus libertades e igualdades, de sus culturas. Estos debates comenzaron a tener unas primeras reacciones políticas. Se pusieron en marcha por primera vez las "políticas de cuotas», con el objetivo de regular la entrada de inmigrantes bajo criterios de población, de nacionalidad y de mercado. En algunos países (Francia) se llegaron incluso a idear políticas para incentivar económicamente a los inmigrantes para que vuelvan a sus países de origen. Al final de esta etapa se produce igualmente una reorientación de prioridades. Los esfuerzos se concentran ahora en políticas de integración, teniendo asumida la necesidad de restringir fuertemente la entrada. En definitiva, conforme avanzamos temporalmente en esta etapa, la inmigración se percibe cada vez con más convicción como problema social, económico y cultural. 
Hoy en día, a pesar de querer permanecer en esta lógica, el fenómeno de la inmigración debe abordarse como lo que es, un problema estructural. El inmigrante no es una mercancía que sirve para solventar o agudizar nuestros problemas estructurales, sino que él mismo es un problema para nuestras estructuras. La crisis de ansiedad que viven nuestros Estados no puede ni debe remediarse con discursos utilitaristas, síntomas de fracasos pasados, además de una falta de responsabilidad. ¿Cómo abordar la inmigración como problema estructural? Ante todo, reconociendo que los inmigrantes deben ser, como todo ser humano, portadores de derechos y de obligaciones. Esta ignorancia se ha debido en parte porque se ha percibido siempre al inmigrante como una condición transitoria, o bien como "alguien de fuera», que regresaría a su país de origen, o bien como potenciales ciudadanos, mediante un proceso de desnaturalización de sus orígenes. Esta situación es hoy en día muy diferente. Debemos comenzar por reconocer que los inmigrantes están aquí para quedarse, y que también desean mantener y transmitir sus formas y expectativas de vida tanto en la esfera privada como en algunos aspectos de la esfera pública. Cualquier otra percepción es simplemente autoengañarse. El problema es que nuestras estructuras tienen serias dificultades para admitir este hecho. Nuestras estructuras están faltas de una cultura de recepción, de una ideología multicultural. Nuestra responsabilidad es encontrar respuestas al desafío histórico que nos plantea la inmigración, revisar y readaptar nuestras estructuras sociales y políticas para que los inmigrantes, al relacionarse con nuestras instituciones, se sientan políticamente cómodos. Sobre cuestiones de inmigración nunca debemos olvidar que si desean venir aquí es porque, de alguna forma, nuestra riqueza económica y Estado de bienestar funciona gracias a la situación de subdesarrollo que padecen sus países de procedencia. Nuestra calidad de vida, nuestra comodidad, se debe en gran parte al subdesarrollo de sus países de origen. Introducir criterios de mercado para discriminar selectivamente entre ellos para permitirles o no el acceso no es sólo injusto, sino pura hipocresía de nuestra sociedad occidental. Tomar la cuestión de la inmigración en serio implica, en primer lugar, reconocer que es un problema estructural.

\section{REFERENCIAS}

Albrow, M. (1996): The global age, Cambridge: Polity Press.

Aragón Bombín, R., y ChOzas Pedrero, J. (1993): La regularización de inmigrantes durante 1991-1992, Madrid: Ministerio de Trabajo y Seguridad Social.

AXtMann, R. (1996): Liberal democracy into the twenty-first century, Manchester: Manchester University Press.

BALIBAR, E. (1992): Les frontières de la démocratie, Paris: La Découverte/essais.

Barber, B. (1996): «La cultura global de McWorld», en J. Rubio Carracedo y J. M. Rosales (eds.), La justicia de los ciudadanos, Málaga: Contrastes, pp. 31-48.

Barry, B., y Goodin, R. E. (eds.) (1992): Free Movement: ethical issues in the transnational migration of people and of money, Pennsylvania: The Pennsylvania State University Press. 
Beetham, D. (ed.) (1994): Defining and measuring democracy, Londres: Sage Publications, pp. 199-221.

Biglino Campos, P. (coord.) (1998): Ciudadanía y extranjería, Madrid: MacGraw-Hill.

BRUBAKER, W. R. (ed.) (1989): Immigration and the politics of citizenship in Europe and North America, London/Lanham: University Press of America.

CARdONA, (1985): Regimen jurídico laboral de los extranjeros en España, Barcelona: Bosch.

- J. (1989): El trabajador extranjero en España, Barcelona: Bosch.

Carens, J. H. (1987): «Aliens and citizens: the case for open borders», en The Review of Politics, 49/2: 251-273.

- (1989): «Membership and morality: admission to citizenship in liberal democratic states», en Brubaker (ed.): pp. 31-49.

- (1995): «Immigration, welfare, and justice», en W. F. Schwartz (ed.), Justice in immigration, Cambridge University Press, pp. 1-17.

- (1997): "Liberalism and culture», en Constellations, 4/1: 35-47.

CASSESE, A. (1993): Los derechos humanos en el mundo contemporáneo, Barcelona: Ariel.

Estrada Carrillo, V. (1989): Comentarios a la Ley Orgánica y Reglamento de Extranjería, Madrid: Trivium

Ferrer Peña, R. M. a (1989): Los derechos de los extranjeros en España, Madrid: Tecnos.

GraY, J. (1993): "The politics of cultural diversity», en Post-liberalism studies in political thought, Nueva York: Routledge, pp. 253-271.

HAMmar, T. (1989): «State, nation, and dual citizenship», en Brubaker (ed.): cap. 4, pp. 81-95.

Held, D. (1997): Democracy and the global order, Polity Press.

Hollifield, J. F. (1992): Immigrants, markets, and states, Cambridge, MA: Harvard University Press.

IZQUIERDO ESCRIBANO, A. (1992): La inmigración en España 1980-1990, Madrid: Ministerio de Trabajo y Seguridad Social.

- (1996): La inmigración inesperada: la población extranjera en España (1991-1995), Madrid: Editorial Trotta.

KYMLICKA, W. (1989a): «Liberal individualism and liberal neutrality», en Ethics, 99: 883-905.

- (1989b): Liberalism, community and culture, Oxford: Clarendon Press.

- (1995): Multicultural citizenship, Oxford: Clarendon Press.

LARMORE, Ch. (1987): Patterns of moral complexity, Cambridge: Cambridge University Press.

- (1990): «Political liberalism», en Political Theory, 18/3: 339-360.

LAYTON-HENRY, Z. (ed.) (1990): The political rights of migrant workers in Western Europe, London: Sage.

LUCAS, J. de (1994): El desafio de las fronteras, Madrid: Temas de Hoy/Ensayo.

Miller, D. (1995): On nationality, Oxford: Clarendon Press.

OOMEn, T. K. (1997): Citizenship, nationality, and ethnicity, Cambridge: Polity Press.

PareKh, B. (1994): "Cultural diversity and liberal democracy», en D. Beetham (ed.): pp. 199221.

Polo SÁnchez, M. C. (1994): Derechos fundamentales y libertades públicas de los trabajadores extranjeros en España, Madrid: Consejo Económico y Social, Colección Estudios.

Prada, M. A. de (1989): «España, de país de emigración a país de inmigración», en M. A. Roque (ed.), Els moviments humans en el Mediterrani occidental (Simposium Internacional), Barcelona: Institut Català d'Estudis Mediterranis, pp. 209-241.

Ramos Quintana, M. (1985): El trabajo de los extranjeros en España, Madrid: Tecnos.

REQUeJO, F. (1995): «Diferències nacionals i federalisme asimètric», en E. Udina (ed.), Quo Vadis, Catalonia?, Barcelona: Planeta, pp. 240-271.

- (1996): «Pluralismo, justicia y federalismo: una revisión de la ciudadanía democrática en estados plurinacionales», en Revista Internacional de Filosofía Politica, 7/mayo: 93-120.

- (1998a): Zoom politic: Democràcia, federalisme i nacionalisme des d'una Catalunya europea, Barcelona: Proa.

- (1998b): Federalisme, per a què? L'acomodació de la diversitat en democràcies plurinacionals, Barcelona: L'hora del Present. 
ReQueJO, F. (ed.): Pluralisme nacional i legitimitat democràtica, Barcelona: Proa.

SCHNAPPER, D. (1994): La communauté des citoyens: sur l'idée moderne de nation, Gallimard.

SCHWARTZ, W. F. (ed.) (1995): Justice in immigration, Cambridge: Cambridge University Press.

TAYLOR, Ch. (1992): Multiculturalism and "the politics of recognition", Princeton: Princeton University Press.

WALZER, M. (1983): Spheres of Justice: a defense of pluralism and equality, Nueva York: Basic Books.

- (1993): «Exclusion, injustice, and the democratic state», en Dissent, 40: 55-64.

- (1997): On toleration, New Haven/Londres: Yale University Press.

WiEVIORKA, M. (1998): Le racisme, une introduction, Paris: La Découverte.

YounG, I. M. (1990): Justice and the politics of difference, Princeton, N. J.: Princeton University Press.

Zapata, R. (1997): «Ética y política de inmigración», en Revista de Treball Social, 146: 68-87.

- (1998a): "Thinking European citizenship from the perspective of an eventual Euro-foreigner", en U. K. Preuß y F. Requejo (eds.), European Citizenship, multiculturalism and the state, Baden-Baden: Nomos 31, cap. 9, pp. 151-168.

- (1998b): «Ciudadanía europea y extranjería», en Claves, 87: 29-35.

- (1999a): "Ciutadania de la Unió i immigració: balanç institucional i desafiaments normatius», en Àmbits de Política i Sociologia, 13: 10-18.

- (1999b): «¿Necesitamos un nuevo concepto de ciudadanía? Estabilidad democrática y pluralismo cultural», en Revista Internacional de Filosofía Política, 13: 119-149.

- (1999c): «A language of global justice towards foreigners", ponencia presentada en el European Consortium for Political Research - Joint Sessions (Mannheim, 16-31 de marzo).

- (2000a): Ciudadanía, democracia y pluralismo cultural: hacia un nuevo contrato social, Barcelona: Proyecto A Ediciones/Biblioteca Episteme.

- (2000b): "The limits of a multinational Europe: democracy and immigration in the European Union», en F. Requejo (ed.), Democracy and national pluralism (Routledge, en prensa).

ZoLO, D. (1997): Cosmopolis: prospect for world government, Cambridge: Polity Press. 


\begin{abstract}
This article examines from a normative point of view the relationship between justice and alien status, in particular questions regarding the limits of justice in a policy for the status of foreigners. The question I have asked myself is: is it morally fair for states to legitimate their policies regarding the status of foreigners on the grounds of economic criteria? The argument guiding my reasoning is that an alien status policy is fairer when the market does not function as a criterion for orientation.

In the first part, I present as a preliminary the new scenarios of the theoretical political debate around the relationship between the market and justice, in order to define the context of my analysis. In the second part, I give a brief introduction to the principal tensions that cast doubt on the legitimacy of state treatment of foreigners from the point of view of justice, with the intention of presenting the logic of my reasoning. In the third part, I propose open reflection that adds to the earlier statement the variable of the status of foreigners, taking the Spanish law on aliens as a study case. In the final part, I sum up my argument: the need to review the criteria that legitimate immigration policies from the viewpoint of justice in general and criteria based on the market in particular.
\end{abstract}

